\title{
Inequalities for eigenvalues of the buckling problem of arbitrary order
}

\author{
Qing-Ming Cheng, Xuerong Qi, Qiaoling Wang and Changyu Xia
}

\begin{abstract}
This paper studies eigenvalues of the buckling problem of arbitrary order on bounded domains in Euclidean spaces and spheres. We prove universal bounds for the $k$-th eigenvalue in terms of the lower ones independent of the domains. Our results strengthen the recent work in [28] and generalize Cheng-Yang's recent estimates [16] on the buckling eigenvalues of order two to arbitrary order.
\end{abstract}

\section{Introduction}

Let $\Omega$ be a bounded domain with smooth boundary in an $n(\geq 2)$-dimensional Riemannian manifold $M$ and denote by $\Delta$ the Laplace operator acting on functions on $M$. Let $\nu$ be the outward unit normal vector field of $\partial \Omega$ and let us consider the following eigenvalue problems :

$$
\begin{aligned}
& \Delta u=-\lambda u \quad \text { in } \Omega, \quad u=0, \quad \text { on } \partial \Omega, \\
& \Delta^{2} u=-\Lambda \Delta u \text { in } \Omega, \quad u=\frac{\partial u}{\partial \nu}=0, \text { on } \partial \Omega .
\end{aligned}
$$

They are called the fixed membrane problem and the bucking problem, respectively. It should be mentioned that the buckling problem (1.2) has interpretations in physics, that is, it describes the critical buckling load of a clamped plate subjected to a uniform compressive force around its boundary. Let

$$
\begin{aligned}
& 0<\lambda_{1}<\lambda_{2} \leq \lambda_{3} \leq \cdots \\
& 0<\Lambda_{1} \leq \Lambda_{2} \leq \Lambda_{3} \leq \cdots
\end{aligned}
$$

denote the successive eigenvalues for (1.1) and (1.2), respectively. Here each eigenvalue is repeated according to its multiplicity. An important theme of geometric analysis is to estimate these (and other) eigenvalues. When $\Omega$ is a bounded domain in an $n$-dimensional Euclidean space $\mathbf{R}^{n}$, Payne, Pólya and Weinberger (cf. [30],[31]) proved the bound

$$
\lambda_{k+1}-\lambda_{k} \leq \frac{4}{k n} \sum_{i=1}^{k} \lambda_{i}, \quad k=1,2, \cdots .
$$

Inequality of this type is called a universal inequality since it does not depend on $\Omega$.

On the other hand, Payne, Pólya and Weinberger also studied eigenvalues of the buckling problem (1.2) for bounded domains in $\mathbf{R}^{n}$ and proved (cf. [30],[31])

$$
\Lambda_{2} / \Lambda_{1}<3 \quad \text { for } \Omega \subset \mathbf{R}^{2} .
$$

For $\Omega \subset \mathbf{R}^{n}$, this reads

$$
\Lambda_{2} / \Lambda_{1}<1+4 / n \text {. }
$$

2000 Mathematics Subject Classification : 35P15, 53C20, 53C42, 58G25

Key words and phrases: Universal inequality for eigenvalues, the buckling problem of arbitrary order, Euclidean space, sphere. 
Furthermore, Payne, Pólya and Weinberger proposed the following

Problem 1 (cf. [30],[31]). Can one obtain a universal inequality for the eigenvalues of the buckling problem (1.2) on a bounded domain in $\mathbf{R}^{n}$ which is similar to the universal inequality (1.3) for the eigenvalues of the fixed membrane problem (1.1)?

With respect to the above problem, Hile and Yeh [26] obtained

$$
\frac{\Lambda_{2}}{\Lambda_{1}} \leq \frac{n^{2}+8 n+20}{(n+2)^{2}} \quad \text { for } \Omega \subset \mathbf{R}^{n} .
$$

Ashbaugh [1] proved :

$$
\sum_{i=1}^{n} \Lambda_{i+1} \leq(n+4) \Lambda_{1}
$$

This inequality has been improved to the following form in [29]:

$$
\sum_{i=1}^{n} \Lambda_{i+1}+\frac{4\left(\Lambda_{2}-\Lambda_{1}\right)}{n+4} \leq(n+4) \Lambda_{1}
$$

By introducing a new method of constructing trial functions, Cheng and Yang [11] obtained the following universal inequality and thus solved the above problem:

$$
\sum_{i=1}^{k}\left(\Lambda_{k+1}-\Lambda_{i}\right)^{2} \leq \frac{4(n+2)}{n^{2}} \sum_{i=1}^{k}\left(\Lambda_{k+1}-\Lambda_{i}\right) \Lambda_{i}
$$

Recently, Cheng-Yang [16] have proved the following inequality:

$$
n \sum_{i=1}^{k}\left(\Lambda_{k+1}-\Lambda_{i}\right)^{2} \leq\left(n+\frac{4}{3}\right) \sum_{i=1}^{k} \delta_{i}\left(\Lambda_{k+1}-\Lambda_{i}\right)^{2}+\sum_{i=1}^{k} \frac{1}{\delta_{i}}\left(\Lambda_{k+1}-\Lambda_{i}\right)^{2} \Lambda_{i},
$$

where $\left\{\delta_{i}\right\}_{i=1}^{k}$ is any positive non-increasing monotone sequence. Taking

$$
\delta_{i}=\sqrt{\frac{\sum_{i=1}^{k}\left(\Lambda_{k+1}-\Lambda_{i}\right)^{2} \Lambda_{i}}{\left(n+\frac{4}{3}\right) \sum_{i=1}^{k}\left(\Lambda_{k+1}-\Lambda_{i}\right)^{2}}}, i=1, \cdots, k
$$

in (1.7), Cheng-Yang have obtained

$$
\sum_{i=1}^{k}\left(\Lambda_{k+1}-\Lambda_{i}\right)^{2} \leq \frac{4\left(n+\frac{4}{3}\right)}{n^{2}} \sum_{i=1}^{k}\left(\Lambda_{k+1}-\Lambda_{i}\right) \Lambda_{i},
$$

which is stronger than (1.6).

It has been proved in [34] that for the problem (1.2) if $\Omega$ is a domain in an $n$-dimensional unit sphere $S^{n}$, then we have

$$
\begin{aligned}
& 2 \sum_{i=1}^{k}\left(\Lambda_{k+1}-\Lambda_{i}\right)^{2} \\
\leq & \sum_{i=1}^{k}\left(\Lambda_{k+1}-\Lambda_{i}\right)^{2}\left(\delta \Lambda_{i}+\frac{\delta^{2}\left(\Lambda_{i}-(n-2)\right)}{4\left(\delta \Lambda_{i}+n-2\right)}\right)+\frac{1}{\delta} \sum_{i=1}^{k}\left(\Lambda_{k+1}-\Lambda_{i}\right)\left(\Lambda_{i}+\frac{(n-2)^{2}}{4}\right),
\end{aligned}
$$


where $\delta$ is any positive constant. This inequality has been improved recently in [16] and [28], respectively.

In this paper, we will investigate eigenvalues of the buckling problem of arbitrary order:

$$
\left\{\begin{array}{cl}
(-\Delta)^{l} u=-\Lambda \Delta u, & \text { in } \Omega, \\
u=\frac{\partial u}{\partial \nu}=\cdots=\frac{\partial^{l-1} u}{\partial \nu^{l-1}}=0, & \text { on } \partial \Omega,
\end{array}\right.
$$

where $\Omega$ is a bounded domain in a Euclidean space or a unit sphere and $l$ is any integer no less than 2 . Yang type inequalities for eigenvalues of the problem (1.10) have been obtained recently in [28]. In this paper, we prove :

Theorem 1.1. Let $\Lambda_{i}$ be the $i$-th eigenvalue of the buckling problem (1.10), where $\Omega$ is a bounded domain with smooth boundary in $\mathbf{R}^{n}$. Then for any positive non-increasing monotone sequence $\left\{\delta_{i}\right\}_{i=1}^{k}$, we have

$$
\begin{aligned}
& n \sum_{i=1}^{k}\left(\Lambda_{k+1}-\Lambda_{i}\right)^{2} \\
\leq & \sum_{i=1}^{k} \delta_{i}\left(\Lambda_{k+1}-\Lambda_{i}\right)^{2}\left(2 l^{2}+\left(n-\frac{14}{3}\right) l+\frac{8}{3}-n\right) \Lambda_{i}^{(l-2) /(l-1)}+\sum_{i=1}^{k} \frac{1}{\delta_{i}}\left(\Lambda_{k+1}-\Lambda_{i}\right) \Lambda_{i}^{1 /(l-1)} .
\end{aligned}
$$

Remark 1.1. When $l=2$, (1.11) becomes Cheng-Yang's inequality (1.7).

Remark 1.2. Taking

$$
\delta_{1}=\delta_{2}=\cdots \delta_{k}=\left\{\frac{\sum_{i=1}^{k}\left(\Lambda_{k+1}-\Lambda_{i}\right) \Lambda_{i}^{1 /(l-1)}}{\left(2 l^{2}+\left(n-\frac{14}{3}\right) l+\frac{8}{3}-n\right) \sum_{i=1}^{k}\left(\Lambda_{k+1}-\Lambda_{i}\right)^{2} \Lambda_{i}^{(l-2) /(l-1)}}\right\}^{1 / 2}
$$

in (1.11), we have

$$
\begin{aligned}
& \sum_{i=1}^{k}\left(\Lambda_{k+1}-\Lambda_{i}\right)^{2} \\
& \leq \frac{2\left(2 l^{2}+\left(n-\frac{14}{3}\right) l+\frac{8}{3}-n\right)^{1 / 2}}{n}\left\{\sum_{i=1}^{k}\left(\Lambda_{k+1}-\Lambda_{i}\right)^{2} \Lambda_{i}^{(l-2) /(l-1)}\right\}^{1 / 2}\left\{\sum_{i=1}^{k}\left(\Lambda_{k+1}-\Lambda_{i}\right) \Lambda_{i}^{1 /(l-1)}\right\}^{1 / 2},
\end{aligned}
$$

which improves the inequality (1.13) in [28]. From (1.12), we can obtain a quadratic inequality about $\Lambda_{1}, \cdots, \Lambda_{k+1}$.

Corollary 1.1. For any $k \geq 1$, the first $k+1$ eigenvalues of the buckling problem (1.10) with $\Omega \subset \mathbf{R}^{n}$ satisfy the following inequality

$$
\sum_{i=1}^{k}\left(\Lambda_{k+1}-\Lambda_{i}\right)^{2} \leq \frac{4\left(2 l^{2}+\left(n-\frac{14}{3}\right) l+\frac{8}{3}-n\right)}{n^{2}} \sum_{i=1}^{k}\left(\Lambda_{k+1}-\Lambda_{i}\right) \Lambda_{i} .
$$

Furthermore, we prove the following universal inequality for eigenvalues of the buckling problem of arbitrary order on spherical domains.

Theorem 1.2. Let $l \geq 2$ and let $\Lambda_{i}$ be the $i$-th eigenvalue of the buckling problem:

$$
\left\{\begin{array}{cc}
(-\Delta)^{l} u=-\Lambda \Delta u, & \text { in } \Omega \\
u=\frac{\partial u}{\partial \nu}=\cdots=\frac{\partial^{l-1} u}{\partial \nu^{l-1}}=0, & \text { on } \partial \Omega
\end{array}\right.
$$


where $\Omega$ is a domain with smooth boundary in $S^{n}$. For each $q=1, \cdots$, define the polynomials $\Phi_{q}$ inductively by

$$
\begin{gathered}
\Phi_{1}(t)=t-1, \Phi_{2}(t)=t^{2}-(n+5) t-(n-2), \\
\Phi_{q}(t)=(2 t-2) \Phi_{q-1}(t)-\left(t^{2}+2 t-n(n-2)\right) \Phi_{q-2}(t), \quad q=3, \cdots .
\end{gathered}
$$

Set

$$
\Phi_{l-1}(t)=t^{l-1}-a_{l-2} t^{l-2}+\cdots+(-1)^{l-2} a_{1} t-(n-2)^{l-2} .
$$

Then for any positive integer $k$ and any positive non-increasing monotone sequence $\left\{\delta_{i}\right\}_{i=1}^{k}$, we have

$$
\begin{aligned}
& \sum_{i=1}^{k}\left(\Lambda_{k+1}-\Lambda_{i}\right)^{2}\left(2+\frac{n-2}{\Lambda_{i}^{1 /(l-1)}-(n-2)}\right) \\
\leq & \sum_{i=1}^{k}\left(\Lambda_{k+1}-\Lambda_{i}\right)^{2} \delta_{i} S_{i}+\sum_{i=1}^{k} \frac{\left(\Lambda_{k+1}-\Lambda_{i}\right)}{\delta_{i}}\left(\Lambda_{i}^{1 /(l-1)}+\frac{(n-2)^{2}}{4}\right),
\end{aligned}
$$

where

$$
S_{i}=\Lambda_{i}\left(1-\frac{1}{\Lambda_{i}^{1 /(l-1)}-(n-2)}\right)+(-1)^{l}(n-2)^{l-2}+\sum_{j=1}^{l-2} a_{j}^{+} \Lambda_{i}^{j /(l-1)}
$$

with $a_{j}^{+}=\max \left\{a_{j}, 0\right\}$ and when $l=2$ we use the convention that $\sum_{j=1}^{l-2} a_{j}^{+} \Lambda_{i}^{j /(l-1)}=0$.

Remark 1.3. When $l=2,(1.18)$ is stronger than (1.9) and it has been proved by Cheng-Yang in [16].

Remark 1.4. Universal inequalities for eigenvalues of various elliptic operators have been studied extensively in recent years. For the developments in this direction, we refer to [1-23], [25-30], [33-40] and the references therein.

Acknowledgement. The research of the first author is partially supported by a Grant-in-Aid for Scientific Research from JSPS, the research of the third author is partially supported by CNPq and the main part of this paper has been done while the fourth author visited to Department of Mathematics, Saga University as a fellowship of JSPS. This author would like to express his gratitude to JSPS for finance support and to Professor Qing-Ming Cheng and Saga University for the worm hospitality.

\section{Proofs of the Results}

First we recall a method of constructing trial functions developed by Cheng-Yang (cf. [11], [28], [34]). Let $M$ be an $n$-dimensional complete submanifold in $\mathbf{R}^{m}$. Denote by $\langle$,$\rangle the canonical metric on \mathbf{R}^{m}$ as well as that induced on $M$. Denote by $\Delta$ and $\nabla$ the Laplacian and the gradient operator of $M$, respectively. Let $\Omega$ be a bounded domain with smooth boundary in $M$ and let $\nu$ be the outward unit normal vector field of $\partial \Omega$. For functions $f$ and $g$ on $\Omega$, the Dirichlet inner product $(f, g)_{D}$ of $f$ and $g$ is given by

$$
(f, g)_{D}=\int_{\Omega}\langle\nabla f, \nabla g\rangle .
$$

The Dirichlet norm of a function $f$ is defined by

$$
\|f\|_{D}=\left\{(f, f)_{D}\right\}^{1 / 2}=\left(\int_{\Omega}|\nabla f|^{2}\right)^{1 / 2} .
$$


Consider the eigenvalue problem

$$
\left\{\begin{array}{cc}
(-\Delta)^{l} u=-\Lambda \Delta u, & \text { in } \Omega, \\
u=\frac{\partial u}{\partial \nu}=\cdots=\frac{\partial^{l-1} u}{\partial \nu^{l-1}}=0, & \text { on } \partial \Omega .
\end{array}\right.
$$

Let

$$
0<\Lambda_{1} \leq \Lambda_{2} \leq \Lambda_{3} \leq \cdots
$$

denote the successive eigenvalues, where each eigenvalue is repeated according to its multiplicity.

Let $u_{i}$ be the $i$-th orthonormal eigenfunction of the problem (2.1) corresponding to the eigenvalue $\Lambda_{i}$, $i=1,2, \cdots$, that is,

$$
\left\{\begin{array}{l}
(-\Delta)^{l} u_{i}=-\Lambda_{i} \Delta u_{i}, \text { in } \Omega, \\
u_{i}=\frac{\partial u_{i}}{\partial \nu}=\cdots=\frac{\partial^{l-1} u_{i}}{\partial \nu^{l-1}}=0, \text { on } \partial \Omega \\
\left(u_{i}, u_{j}\right)_{D}=\int_{\Omega}\left\langle\nabla u_{i}, \nabla u_{j}\right\rangle=\delta_{i j}, \quad \forall i, j .
\end{array}\right.
$$

For $k=1, \cdots, l$, let $\nabla^{k}$ denote the $k$-th covariant derivative operator on $M$, defined in the usual weak sense. For a function $f$ on $\Omega$, the squared norm of $\nabla^{k} f$ is defined as (cf. [24])

$$
\left|\nabla^{k} f\right|^{2}=\sum_{i_{1}, \cdots, i_{k}=1}^{n}\left(\nabla^{k} f\left(e_{i_{1}}, \cdots, e_{i_{k}}\right)\right)^{2},
$$

where $e_{1}, \cdots, e_{n}$ are orthonormal vector fields locally defined on $\Omega$. Define the Sobolev space $H_{l}^{2}(\Omega)$ by

$$
H_{l}^{2}(\Omega)=\left\{f: f,|\nabla f|, \cdots,\left|\nabla^{l} f\right| \in L^{2}(\Omega)\right\} .
$$

Then $H_{l}^{2}(\Omega)$ is a Hilbert space with respect to the norm $\|\cdot\|_{l, 2}$ :

$$
\|f\|_{l, 2}=\left(\int_{\Omega}\left(\sum_{k=0}^{l}\left|\nabla^{k} f\right|^{2}\right)\right)^{1 / 2} .
$$

Consider the subspace $H_{l, D}^{2}(\Omega)$ of $H_{l}^{2}(\Omega)$ defined by

$$
H_{l, D}^{2}(\Omega)=\left\{f \in H_{l}^{2}(\Omega):\left.f\right|_{\partial \Omega}=\left.\frac{\partial f}{\partial \nu}\right|_{\partial \Omega}=\left.\cdots \frac{\partial^{l-1} f}{\partial \nu^{l-1}}\right|_{\partial \Omega}=0\right\} .
$$

The operator $(-\Delta)^{l}$ defines a self-adjoint operator acting on $H_{l, D}^{2}(\Omega)$ with discrete eigenvalues $0<\Lambda_{1} \leq$ $\cdots \leq \Lambda_{k} \leq \cdots$ for the buckling problem (2.1) and the eigenfunctions $\left\{u_{i}\right\}_{i=1}^{\infty}$ defined in (2.2) form a complete orthonormal basis for the Hilbert space $H_{l, D}^{2}(\Omega)$. If $\phi \in H_{l, D}^{2}(\Omega)$ satisfies $\left(\phi, u_{j}\right)_{D}=0, \forall j=$ $1,2, \cdots, k$, then the Rayleigh-Ritz inequality tells us that

$$
\Lambda_{k+1}\|\phi\|_{D}^{2} \leq \int_{\Omega} \phi(-\Delta)^{l} \phi .
$$

For vector-valued functions $F=\left(f_{1}, f_{2}, \cdots, f_{m}\right), G=\left(g_{1}, g_{2}, \cdots, g_{m}\right): \Omega \rightarrow \mathbf{R}^{m}$, we define an inner product $(F, G)$ by

$$
(F, G) \equiv \int_{\Omega}\langle F, G\rangle=\int_{\Omega} \sum_{\alpha=1}^{m} f_{\alpha} g_{\alpha}
$$

The norm of $F$ is given by

$$
\|F\|=(F, F)^{1 / 2}=\left\{\int_{\Omega} \sum_{\alpha=1}^{m} f_{\alpha}^{2}\right\}^{1 / 2} .
$$


Let $\mathbf{H}_{1}^{2}(\Omega)$ be the Hilbert space of vector-valued functions given by

$$
\mathbf{H}_{1}^{2}(\Omega)=\left\{F=\left(f_{1}, \cdots, f_{m}\right): \Omega \rightarrow \mathbf{R}^{m} ; f_{\alpha},\left|\nabla f_{\alpha}\right| \in L^{2}(\Omega), \text { for } \alpha=1, \cdots, m\right\}
$$

with norm

$$
\|F\|_{1}=\left(\|F\|^{2}+\int_{\Omega} \sum_{\alpha=1}^{m}\left|\nabla f_{\alpha}\right|^{2}\right)^{1 / 2} .
$$

Observe that a vector field on $\Omega$ can be regarded as a vector-valued function from $\Omega$ to $\mathbf{R}^{m}$. Let $\mathbf{H}_{1, D}^{2}(\Omega) \subset \mathbf{H}_{1}^{2}(\Omega)$ be a subspace of $\mathbf{H}_{1}^{2}(\Omega)$ spanned by the vector-valued functions $\left\{\nabla u_{i}\right\}_{i=1}^{\infty}$, which form a complete orthonormal basis of $\mathbf{H}_{1, D}^{2}(\Omega)$. For any $f \in H_{l, D}^{2}(\Omega)$, we have $\nabla f \in \mathbf{H}_{1, D}^{2}(\Omega)$ and for any $X \in \mathbf{H}_{1, D}^{2}(\Omega)$, there exists a function $f \in H_{l, D}^{2}(\Omega)$ such that $X=\nabla f$.

Lemma 2.1. (cf. [28],[29]) Let $u_{i}$ and $\Lambda_{i}, i=1,2, \cdots$, be as in (2.2), then

$$
0 \leq \int_{\Omega} u_{i}(-\Delta)^{k} u_{i} \leq \Lambda_{i}^{(k-1) /(l-1)}, \quad k=1, \cdots, l-1 .
$$

We are now ready to prove the main results in this paper.

Proof of Theorem 1.1. With the notations as above, we consider now the special case that $\Omega$ is a bounded domain in $\mathbf{R}^{n}$. Denote by $x_{1}, \cdots, x_{n}$ the coordinate functions of $\mathbf{R}^{n}$ and let us decompose the vectorvalued functions $x_{\alpha} \nabla u_{i}$ as

$$
x_{\alpha} \nabla u_{i}=\nabla h_{\alpha i}+W_{\alpha i},
$$

where $h_{\alpha i} \in H_{l, D}^{2}(\Omega), \nabla h_{\alpha i}$ is the projection of $x_{\alpha} \nabla u_{i}$ in $\mathbf{H}_{1, D}^{2}(\Omega)$ and $W_{\alpha i} \perp \mathbf{H}_{1, D}^{2}(\Omega)$. Thus we have

$$
\left.W_{\alpha i}\right|_{\partial \Omega}=0, \text { and }\left(W_{\alpha i}, \nabla u\right)=\int_{\Omega}\left\langle W_{\alpha i}, \nabla u\right\rangle=0, \text { for any } u \in H_{l, D}^{2}(\Omega)
$$

and from the discussions in [11] and [34] we know that

$$
\operatorname{div} W_{\alpha i}=0,
$$

where for a vector field $Z$ on $\Omega$, div $Z$ denotes the divergence of $Z$.

For each $\alpha=1, \cdots, n, i=1, \cdots, k$, consider the functions $\phi_{\alpha i}: \Omega \rightarrow \mathbf{R}$, given by

$$
\phi_{\alpha i}=h_{\alpha i}-\sum_{j=1}^{k} a_{\alpha i j} u_{j},
$$

where

$$
a_{\alpha i j}=\int_{\Omega} x_{\alpha}\left\langle\nabla u_{i}, \nabla u_{j}\right\rangle=a_{\alpha j i}
$$

We have

$$
\begin{gathered}
\left.\phi_{\alpha i}\right|_{\partial \Omega}=\left.\frac{\partial \phi_{\alpha i}}{\partial \nu}\right|_{\partial \Omega}=\left.\cdots \frac{\partial^{l-1} \phi_{\alpha i}}{\partial \nu^{l-1}}\right|_{\partial \Omega}=0, \\
\left(\phi_{\alpha i}, u_{j}\right)_{D}=\int_{\Omega}\left\langle\nabla \phi_{\alpha i}, \nabla u_{j}\right\rangle=0, \quad \forall j=1, \cdots, k .
\end{gathered}
$$


It then follows from the Rayleigh-Ritz inequality for $\Lambda_{k+1}$ that

$$
\Lambda_{k+1} \int_{\Omega}\left|\nabla \phi_{\alpha i}\right|^{2} \leq \int_{\Omega} \phi_{\alpha i}(-\Delta)^{l} \phi_{\alpha i}, \quad \forall \alpha=1, \cdots, n, \quad i=1, \cdots, k .
$$

After some calculations, we have (cf. (2.36) in [28])

$$
\begin{aligned}
\int_{\Omega} \phi_{\alpha i}(-\Delta)^{l} \phi_{\alpha i}= & \int_{\Omega}(-1)^{l}\left\{(-l+1) u_{i} \Delta^{l-1} u_{i}+\left(2 l^{2}-4 l+3\right)\left(\Delta^{l-2} u_{i}\right)_{, \alpha} u_{i, \alpha}\right\} \\
& +\Lambda_{i}\left\{\int_{\Omega} x_{\alpha}^{2}\left|\nabla u_{i}\right|^{2}-\int_{\Omega} u_{i}^{2}\right\}-\sum_{j=1}^{k} \Lambda_{j} a_{\alpha i j}^{2} .
\end{aligned}
$$

It is easy to see that

$$
\left\|x_{\alpha} \nabla u_{i}\right\|^{2}=\left\|\nabla h_{\alpha i}\right\|^{2}+\left\|W_{\alpha i}\right\|^{2}, \quad\left\|\nabla h_{\alpha i}\right\|^{2}=\left\|\nabla \phi_{\alpha i}\right\|^{2}+\sum_{j=1}^{k} a_{\alpha i j}^{2},
$$

where for a vector field $Z$ on $\Omega,\|Z\|^{2}=\int_{\Omega}|Z|^{2}$. Combining (2.14)-(2.16), we infer

$$
\begin{aligned}
\left(\Lambda_{k+1}-\Lambda_{i}\right)\left\|\nabla \phi_{\alpha i}\right\|^{2} \leq & \int_{\Omega}(-1)^{l}\left\{(-l+1) u_{i} \Delta^{l-1} u_{i}+\left(2 l^{2}-4 l+3\right)\left(\Delta^{l-2} u_{i}\right)_{, \alpha} u_{i, \alpha}\right\} \\
& -\Lambda_{i}\left(\left\|u_{i}\right\|^{2}-\left\|W_{\alpha i}\right\|^{2}\right)+\sum_{j=1}^{k}\left(\Lambda_{i}-\Lambda_{j}\right) a_{\alpha i j}^{2}
\end{aligned}
$$

Observe that $\nabla\left(x_{\alpha} u_{i}\right)=u_{i} \nabla x_{\alpha}+x_{\alpha} \nabla u_{i} \in \mathbf{H}_{1, D}^{2}(\Omega)$. Set $y_{\alpha i}=x_{\alpha} u_{i}-h_{\alpha i}$; then

$$
u_{i} \nabla x_{\alpha}=\nabla y_{\alpha i}-W_{\alpha i} .
$$

and so

$$
\left\|u_{i}\right\|^{2}=\left\|u_{i} \nabla x_{\alpha}\right\|^{2}=\left\|W_{\alpha i}\right\|^{2}+\left\|\nabla y_{\alpha i}\right\|^{2} .
$$

Substituting (2.18) into (2.17), we get

$$
\begin{aligned}
& \left(\Lambda_{k+1}-\Lambda_{i}\right)\left\|\nabla \phi_{\alpha i}\right\|^{2} \\
\leq & \int_{\Omega}(-1)^{l}\left\{(-l+1) u_{i} \Delta^{l-1} u_{i}+\left(2 l^{2}-4 l+3\right)\left(\Delta^{l-2} u_{i}\right)_{, \alpha} u_{i, \alpha}\right\} \\
& -\Lambda_{i}\left\|\nabla y_{\alpha i}\right\|^{2}+\sum_{j=1}^{k}\left(\Lambda_{i}-\Lambda_{j}\right) a_{\alpha i j}^{2} .
\end{aligned}
$$

Summing on $\alpha$ from 1 to $n$, we have

$$
\begin{aligned}
& \left(\Lambda_{k+1}-\Lambda_{i}\right) \sum_{\alpha=1}^{n}\left\|\nabla \phi_{\alpha i}\right\|^{2} \\
\leq & \int_{\Omega}(-1)^{l}\left\{n(-l+1) u_{i} \Delta^{l-1} u_{i}+\left(2 l^{2}-4 l+3\right)\left\langle\nabla\left(\Delta^{l-2} u_{i}\right), \nabla u_{i}\right\rangle\right\} \\
& -\Lambda_{i} \sum_{\alpha=1}^{n}\left\|\nabla y_{\alpha i}\right\|^{2}+\sum_{\alpha=1}^{n} \sum_{j=1}^{k}\left(\Lambda_{i}-\Lambda_{j}\right) a_{\alpha i j}^{2} \\
= & \left(2 l^{2}+(n-4) l+3-n\right) \int_{\Omega} u_{i}(-\Delta)^{l-1} u_{i}-\Lambda_{i} \sum_{\alpha=1}^{n}\left\|\nabla y_{\alpha i}\right\|^{2}+\sum_{\alpha=1}^{n} \sum_{j=1}^{k}\left(\Lambda_{i}-\Lambda_{j}\right) a_{\alpha i j}^{2} .
\end{aligned}
$$


Using the divergence theorem, one can show that (cf. [11], [28])

$$
-2 \int_{\Omega} x_{\alpha}\left\langle\nabla u_{i}, \nabla\left\langle\nabla u_{i}, \nabla x_{\alpha}\right\rangle\right\rangle=1
$$

Set

$$
d_{\alpha i j}=\int_{\Omega}\left\langle\nabla\left\langle\nabla u_{i}, \nabla x_{\alpha}\right\rangle, \nabla u_{j}\right\rangle,
$$

then $d_{\alpha i j}=-d_{\alpha j i}$ and we have from (2.7), (2.8), (2.10) and (2.20) that

$$
\begin{aligned}
1 & =-2 \int_{\Omega}\left\langle\nabla h_{\alpha i}, \nabla\left\langle\nabla u_{i}, \nabla x_{\alpha}\right\rangle\right\rangle \\
& =-2 \int_{\Omega}\left\langle\nabla \phi_{\alpha i}, \nabla\left\langle\nabla u_{i}, \nabla x_{\alpha}\right\rangle\right\rangle-2 \sum_{j=1}^{k} a_{\alpha i j} d_{\alpha i j} .
\end{aligned}
$$

Thus, we have

$$
\begin{aligned}
& \left(\Lambda_{k+1}-\Lambda_{i}\right)^{2}\left(1+2 \sum_{j=1}^{k} a_{\alpha i j} d_{\alpha i j}\right) \\
= & \left(\Lambda_{k+1}-\Lambda_{i}\right)^{2}\left(-2 \nabla \phi_{\alpha i}, \nabla u_{i, \alpha}-\sum_{j=1}^{k} d_{\alpha i j} \nabla u_{j}\right) \\
\leq & \delta_{i}\left(\Lambda_{k+1}-\Lambda_{i}\right)^{3}\left\|\nabla \phi_{\alpha i}\right\|^{2}+\frac{1}{\delta_{i}}\left(\Lambda_{k+1}-\Lambda_{i}\right)\left(\left\|\nabla u_{i, \alpha}\right\|^{2}-\sum_{j=1}^{k} d_{\alpha i j}^{2}\right),
\end{aligned}
$$

where $u_{i, \alpha}=\left\langle\nabla u_{i}, \nabla x_{\alpha}\right\rangle$. Summing on $\alpha$ from 1 to $n$, we have by using (2.19) that

$$
\begin{aligned}
& \left(\Lambda_{k+1}-\Lambda_{i}\right)^{2}\left(n+2 \sum_{\alpha=1}^{n} \sum_{j=1}^{k} a_{\alpha i j} d_{\alpha i j}\right) \\
\leq & \delta_{i}\left(\Lambda_{k+1}-\Lambda_{i}\right)^{2}\left(\left(2 l^{2}+(n-4) l+3-n\right) \int_{\Omega} u_{i}(-\Delta)^{l-1} u_{i}-\Lambda_{i} \sum_{\alpha=1}^{n}\left\|\nabla y_{\alpha i}\right\|^{2}\right. \\
& \left.+\sum_{\alpha=1}^{n} \sum_{j=1}^{k}\left(\Lambda_{i}-\Lambda_{j}\right) a_{\alpha i j}^{2}\right)+\frac{1}{\delta_{i}}\left(\Lambda_{k+1}-\Lambda_{i}\right)\left(\sum_{\alpha=1}^{n}\left\|\nabla u_{i, \alpha}\right\|^{2}-\sum_{\alpha=1}^{n} \sum_{j=1}^{k} d_{\alpha i j}^{2}\right) .
\end{aligned}
$$

Summing on $i$ from 1 to $k$ and noticing the fact that $a_{\alpha i j}=a_{\alpha j i}, d_{\alpha i j}=-d_{\alpha j i}$, one gets

$$
\begin{aligned}
& n \sum_{i=1}^{k}\left(\Lambda_{k+1}-\Lambda_{i}\right)^{2}-2 \sum_{\alpha=1}^{n} \sum_{i, j=1}^{k}\left(\Lambda_{k+1}-\Lambda_{i}\right)\left(\Lambda_{i}-\Lambda_{j}\right) a_{\alpha i j} d_{\alpha i j} \\
\leq & \sum_{i=1}^{k} \delta_{i}\left(\Lambda_{k+1}-\Lambda_{i}\right)^{2}\left(\left(2 l^{2}+(n-4) l+3-n\right) \int_{\Omega} u_{i}(-\Delta)^{l-1} u_{i}-\Lambda_{i} \sum_{\alpha=1}^{n}\left\|\nabla y_{\alpha i}\right\|^{2}\right) \\
& -\sum_{\alpha=1}^{n} \sum_{i, j=1}^{k} \delta_{i}\left(\Lambda_{k+1}-\Lambda_{i}\right)\left(\Lambda_{i}-\Lambda_{j}\right)^{2} a_{\alpha i j}^{2}-\sum_{\alpha=1}^{n} \sum_{i, j=1}^{k} \frac{1}{\delta_{i}}\left(\Lambda_{k+1}-\Lambda_{i}\right) d_{\alpha i j}^{2} \\
& +\sum_{i=1}^{n} \frac{1}{\delta_{i}}\left(\Lambda_{k+1}-\Lambda_{i}\right) \sum_{\alpha=1}^{n}\left\|\nabla u_{i, \alpha}\right\|^{2} \\
& +\sum_{\alpha=1}^{n} \sum_{i, j=1}^{k} \delta_{i}\left(\Lambda_{k+1}-\Lambda_{i}\right)\left(\Lambda_{i}-\Lambda_{j}\right)^{2} a_{\alpha i j}^{2}+\sum_{\alpha=1}^{n} \sum_{i, j=1}^{k} \delta_{i}\left(\Lambda_{k+1}-\Lambda_{i}\right)^{2}\left(\Lambda_{i}-\Lambda_{j}\right) a_{\alpha i j}^{2} .
\end{aligned}
$$


Since $\left\{\delta_{i}\right\}_{i=1}^{k}$ is a non-increasing monotone sequence, we have

$$
\begin{aligned}
& \sum_{\alpha=1}^{n} \sum_{i, j=1}^{k} \delta_{i}\left(\Lambda_{k+1}-\Lambda_{i}\right)\left(\Lambda_{i}-\Lambda_{j}\right)^{2} a_{\alpha i j}^{2}+\sum_{\alpha=1}^{n} \sum_{i, j=1}^{k} \delta_{i}\left(\Lambda_{k+1}-\Lambda_{i}\right)^{2}\left(\Lambda_{i}-\Lambda_{j}\right) a_{\alpha i j}^{2} \\
= & \frac{1}{2} \sum_{\alpha=1}^{n} \sum_{i, j=1}^{k}\left(\Lambda_{k+1}-\Lambda_{i}\right)\left(\Lambda_{k+1}-\Lambda_{j}\right)\left(\Lambda_{i}-\Lambda_{j}\right)\left(\delta_{i}-\delta_{j}\right) a_{\alpha i j}^{2} \leq 0 .
\end{aligned}
$$

We conclude from (2.22) that

$$
\begin{aligned}
& n \sum_{i=1}^{k}\left(\Lambda_{k+1}-\Lambda_{i}\right)^{2} \\
\leq & \sum_{i=1}^{k} \delta_{i}\left(\Lambda_{k+1}-\Lambda_{i}\right)^{2}\left(\left(2 l^{2}+(n-4) l+3-n\right) \int_{\Omega} u_{i}(-\Delta)^{l-1} u_{i}-\Lambda_{i} \sum_{\alpha=1}^{n}\left\|\nabla y_{\alpha i}\right\|^{2}\right) \\
& +\sum_{i=1}^{n} \frac{1}{\delta_{i}}\left(\Lambda_{k+1}-\Lambda_{i}\right) \sum_{\alpha=1}^{n}\left\|\nabla u_{i, \alpha}\right\|^{2} .
\end{aligned}
$$

It follows from the divergence theorem and Lemma 2.1 that

$$
\begin{aligned}
\sum_{\alpha=1}^{k}\left\|\nabla u_{i, \alpha}\right\|^{2} & =-\int_{\Omega} \sum_{\alpha=1}^{k} u_{i, \alpha} \Delta u_{i, \alpha} \\
& =-\int_{\Omega} \sum_{\alpha=1}^{k} u_{i, \alpha}\left(\Delta u_{i}\right)_{, \alpha} \\
& =\int_{\Omega} \sum_{\alpha=1}^{k} u_{i, \alpha \alpha} \Delta u_{i} \\
& =\int_{\Omega}\left(\Delta u_{i}\right)^{2} \\
& =\int_{\Omega} u_{i} \Delta^{2} u_{i} \\
& \leq \Lambda_{i}^{1 /(l-1)},
\end{aligned}
$$

where $u_{i, \alpha \alpha}=\frac{\partial^{2} u_{i}}{\partial x_{\alpha}^{2}}$. Thus, we have

$$
\begin{aligned}
& n \sum_{i=1}^{k}\left(\Lambda_{k+1}-\Lambda_{i}\right)^{2} \\
\leq & \sum_{i=1}^{k} \delta_{i}\left(\Lambda_{k+1}-\Lambda_{i}\right)^{2}\left(\left(2 l^{2}+(n-4) l+3-n\right) \int_{\Omega} u_{i}(-\Delta)^{l-1} u_{i}-\sum_{\alpha=1}^{n} \Lambda_{i}\left\|\nabla y_{\alpha i}\right\|^{2}\right) \\
& +\sum_{i=1}^{k} \frac{1}{\delta_{i}}\left(\Lambda_{k+1}-\Lambda_{i}\right) \Lambda_{i}^{1 /(l-1)} .
\end{aligned}
$$

Before we can finish the proof of Theorem 1.2, we shall need two lemmas.

Lemma 2.2. For any $i$, we have

$$
(n-2 l-2) \int_{\Omega} u_{i}(-\Delta)^{l-1} u_{i}=n \Lambda_{i}\left\|u_{i}\right\|^{2}-4 \Lambda_{i} \sum_{\alpha=1}^{n}\left\|\nabla y_{\alpha i}\right\|^{2} .
$$


Proof. When $l=2$, the above formula has been proved by Cheng-Yang in [16]. We only consider the case that $l>2$. In this case, we conclude from the boundary condition on $u_{i}$ that $\left.y_{\alpha i}\right|_{\partial \Omega}=\left.\nabla y_{\alpha i}\right|_{\partial \Omega}=$ $\left.\Delta y_{\alpha i}\right|_{\partial \Omega}=0$. Using the divergence theorem, we have

$$
\begin{aligned}
& \int_{\Omega} x_{\alpha} u_{i}\left\langle\nabla x_{\alpha}, \nabla\left(\Delta^{l-1} u_{i}\right)\right\rangle=\int_{\Omega} x_{\alpha} u_{i} \Delta^{l-1}\left\langle\nabla x_{\alpha}, \nabla u_{i}\right\rangle \\
& =\int_{\Omega} \Delta^{l-1}\left(x_{\alpha} u_{i}\right)\left\langle\nabla x_{\alpha}, \nabla u_{i}\right\rangle \\
& =-\int_{\Omega}\left\langle u_{i} \nabla x_{\alpha}, \nabla\left(\Delta^{l-1}\left(x_{\alpha} u_{i}\right)\right)\right\rangle \\
& =-\int_{\Omega}\left\langle\nabla y_{\alpha i}, \nabla\left(\Delta^{l-1}\left(x_{\alpha} u_{i}\right)\right)\right\rangle \\
& =\int_{\Omega} y_{\alpha i} \Delta^{l}\left(x_{\alpha} u_{i}\right) \\
& =\int_{\Omega} y_{\alpha i}\left(2 l\left\langle\nabla\left(\Delta^{l-1} u_{i}\right), \nabla x_{\alpha}\right\rangle+x_{\alpha} \Delta^{l} u_{i}\right) \\
& =-2 l \int_{\Omega} \Delta^{l-1} u_{i}\left\langle\nabla y_{\alpha i}, \nabla x_{\alpha}\right\rangle+\Lambda_{i}(-1)^{l-1} \int_{\Omega} y_{\alpha i} x_{\alpha} \Delta u_{i}, \\
& \int_{\Omega} y_{\alpha i} x_{\alpha} \Delta u_{i}=-\int_{\Omega}\left\langle\nabla y_{\alpha i}, x_{\alpha} \nabla u_{i}\right\rangle-\int_{\Omega} y_{\alpha i}\left\langle\nabla x_{\alpha}, \nabla u_{i}\right\rangle \\
& =-\int_{\Omega}\left\langle\nabla y_{\alpha i}, x_{\alpha} \nabla u_{i}\right\rangle+\int_{\Omega}\left\langle\nabla y_{\alpha i}, u_{i} \nabla x_{\alpha}\right\rangle \\
& =-\int_{\Omega}\left\langle\nabla y_{\alpha i}, x_{\alpha} \nabla u_{i}\right\rangle+\left\|\nabla y_{\alpha i}\right\|^{2} \text {, } \\
& \int_{\Omega}\left\langle\nabla y_{\alpha i}, x_{\alpha} \nabla u_{i}\right\rangle=\int_{\Omega}\left\langle\nabla y_{\alpha i}, \nabla h_{\alpha i}\right\rangle \\
& =\int_{\Omega}\left\langle\nabla y_{\alpha i}, \nabla\left(x_{\alpha} u_{i}\right)-\nabla y_{\alpha i}\right\rangle \\
& =\int_{\Omega}\left\langle\nabla y_{\alpha i}, \nabla\left(x_{\alpha} u_{i}\right)\right\rangle-\left\|\nabla y_{\alpha i}\right\|^{2} \\
& =\int_{\Omega}\left\langle u_{i} \nabla x_{\alpha}, \nabla\left(x_{\alpha} u_{i}\right)\right\rangle-\left\|\nabla y_{\alpha i}\right\|^{2} \\
& =\left\|u_{i}\right\|^{2}+\int_{\Omega}\left\langle u_{i} \nabla x_{\alpha}, x_{\alpha} \nabla u_{i}\right\rangle-\left\|\nabla y_{\alpha i}\right\|^{2} \\
& =\left\|u_{i}\right\|^{2}-\frac{1}{4} \int_{\Omega} u_{i}^{2} \Delta x_{\alpha}^{2}-\left\|\nabla y_{\alpha i}\right\|^{2} \\
& =\frac{1}{2}\left\|u_{i}\right\|^{2}-\left\|\nabla y_{\alpha i}\right\|^{2}
\end{aligned}
$$

and

$$
\begin{aligned}
\int_{\Omega} \Delta^{l-1} u_{i}\left\langle\nabla y_{\alpha i}, \nabla x_{\alpha}\right\rangle & =-\int_{\Omega} y_{\alpha i}\left\langle\nabla\left(\Delta^{l-1} u_{i}\right), \nabla x_{\alpha}\right\rangle \\
& =-\int_{\Omega} y_{\alpha i} \Delta\left\langle\nabla\left(\Delta^{l-2} u_{i}\right), \nabla x_{\alpha}\right\rangle \\
& =\int_{\Omega}\left\langle\nabla y_{\alpha i}, \nabla\left\langle\nabla\left(\Delta^{l-2} u_{i}\right), \nabla x_{\alpha}\right\rangle\right\rangle
\end{aligned}
$$




$$
\begin{aligned}
& =\int_{\Omega}\left\langle u_{i} \nabla x_{\alpha}, \nabla\left\langle\nabla\left(\Delta^{l-2} u_{i}\right), \nabla x_{\alpha}\right\rangle\right\rangle \\
& =-\int_{\Omega}\left\langle\nabla x_{\alpha}, \nabla\left(\Delta^{l-2} u_{i}\right)\right\rangle\left\langle\nabla u_{i}, \nabla x_{\alpha}\right\rangle .
\end{aligned}
$$

It follows from (2.26)-(2.29) that

$$
\begin{aligned}
& \int_{\Omega} x_{\alpha} u_{i}\left\langle\nabla x_{\alpha}, \nabla\left(\Delta^{l-1} u_{i}\right)\right\rangle \\
= & 2 l \int_{\Omega}\left\langle\nabla x_{\alpha}, \nabla\left(\Delta^{l-2} u_{i}\right)\right\rangle\left\langle\nabla u_{i}, \nabla x_{\alpha}\right\rangle+(-1)^{l-1} \Lambda_{i}\left(-\frac{1}{2}\left\|u_{i}\right\|^{2}+2\left\|\nabla y_{\alpha i}\right\|^{2}\right) .
\end{aligned}
$$

Since

$$
\Delta^{l-1}\left(x_{\alpha} u_{i}\right)=2(l-1)\left\langle\nabla\left(\Delta^{l-2} u_{i}\right), \nabla x_{\alpha}\right\rangle+x_{\alpha} \Delta^{l-1} u_{i}
$$

we get

$$
\begin{aligned}
\int_{\Omega} x_{\alpha} u_{i}\left\langle\nabla x_{\alpha}, \nabla\left(\Delta^{l-1} u_{i}\right)\right\rangle & =\int_{\Omega} x_{\alpha} u_{i} \Delta^{l-1}\left\langle\nabla u_{i}, \nabla x_{\alpha}\right\rangle \\
& =\int_{\Omega} \Delta^{l-1}\left(x_{\alpha} u_{i}\right)\left\langle\nabla u_{i}, \nabla x_{\alpha}\right\rangle \\
& =\int_{\Omega}\left(2(l-1)\left\langle\nabla\left(\Delta^{l-2} u_{i}\right), \nabla x_{\alpha}\right\rangle+x_{\alpha} \Delta^{l-1} u_{i}\right)\left\langle\nabla u_{i}, \nabla x_{\alpha}\right\rangle .
\end{aligned}
$$

On the other hand, we have

$$
\int_{\Omega} x_{\alpha} u_{i}\left\langle\nabla x_{\alpha}, \nabla\left(\Delta^{l-1} u_{i}\right)\right\rangle=-\int_{\Omega} \Delta^{l-1} u_{i}\left(u_{i}+x_{\alpha}\left\langle\nabla u_{i}, \nabla x_{\alpha}\right\rangle\right) .
$$

We obtain from (2.31) and (2.32) that

$$
\begin{aligned}
& \int_{\Omega} x_{\alpha} u_{i}\left\langle\nabla x_{\alpha}, \nabla\left(\Delta^{l-1} u_{i}\right)\right\rangle \\
= & \int_{M}\left\{(l-1)\left(\left\langle\nabla\left(\Delta^{l-2} u_{i}\right), \nabla x_{\alpha}\right\rangle\left\langle\nabla u_{i}, \nabla x_{\alpha}\right\rangle-\frac{1}{2} u_{i} \Delta^{l-1} u_{i}\right\} .\right.
\end{aligned}
$$

Combining (2.30) and (2.33), we infer

$$
\begin{aligned}
& \int_{M}\left\{(l-1)\left\langle\nabla x_{\alpha}, \nabla\left(\Delta^{l-2} u_{i}\right)\right\rangle\left\langle\nabla x_{\alpha}, \nabla u_{i}\right\rangle-\frac{1}{2} u_{i} \Delta^{l-1} u_{i}\right\} \\
= & 2 l \int_{\Omega}\left\langle\nabla x_{\alpha}, \nabla\left(\Delta^{l-2} u_{i}\right)\right\rangle\left\langle\nabla u_{i}, \nabla x_{\alpha}\right\rangle+(-1)^{l-1} \Lambda_{i}\left(-\frac{1}{2}\left\|u_{i}\right\|^{2}+2\left\|\nabla y_{\alpha i}\right\|^{2}\right) .
\end{aligned}
$$

Summing on $\alpha$, we get (2.25).

Lemma 2.3. For any $i$, we have

$$
\sum_{\alpha=1}^{n}\left\|W_{\alpha i}\right\|^{2} \geq \frac{n-1}{\Lambda_{i}^{1 /(l-1)}}
$$

Proof. Using the definition of $W_{\alpha i}$ and the divergence theorem and noticing (2.20), we have

$$
\int_{\Omega}\left\langle\nabla x_{\alpha}, W_{\alpha i}\right\rangle \Delta u_{i}
$$




$$
\begin{aligned}
& =-\int_{\Omega}\left\langle\nabla u_{i}, \nabla\left\langle\nabla x_{\alpha}, W_{\alpha i}\right\rangle\right\rangle \\
& =-\int_{\Omega}\left\langle\nabla u_{i}, \nabla\left(\left\langle x_{\alpha} \nabla u_{i}-\nabla h_{\alpha i}, \nabla x_{\alpha}\right\rangle\right)\right\rangle \\
& =-\left\|\left\langle\nabla u_{i}, \nabla x_{\alpha}\right\rangle\right\|^{2}-\int_{\Omega} x_{\alpha}\left\langle\nabla u_{i}, \nabla\left\langle\nabla u_{i}, \nabla x_{\alpha}\right\rangle\right\rangle+\int_{\Omega}\left\langle\nabla u_{i}, \nabla\left\langle\nabla h_{\alpha i}, \nabla x_{\alpha}\right\rangle\right\rangle \\
& =-\left\|\left\langle\nabla u_{i}, \nabla x_{\alpha}\right\rangle\right\|^{2}-\int_{\Omega} x_{\alpha}\left\langle\nabla u_{i}, \nabla\left\langle\nabla u_{i}, \nabla x_{\alpha}\right\rangle\right\rangle-\int_{\Omega} u_{i} \Delta\left\langle\nabla h_{\alpha i}, \nabla x_{\alpha}\right\rangle \\
& =-\left\|\left\langle\nabla u_{i}, \nabla x_{\alpha}\right\rangle\right\|^{2}-\int_{\Omega} x_{\alpha}\left\langle\nabla u_{i}, \nabla\left\langle\nabla u_{i}, \nabla x_{\alpha}\right\rangle\right\rangle-\int_{\Omega} u_{i}\left\langle\nabla\left(\Delta h_{\alpha i}\right), \nabla x_{\alpha}\right\rangle \\
& =-\left\|\left\langle\nabla u_{i}, \nabla x_{\alpha}\right\rangle\right\|^{2}-\int_{\Omega}\left\langle x_{\alpha} \nabla u_{i}, \nabla\left\langle\nabla u_{i}, \nabla x_{\alpha}\right\rangle\right\rangle-\int_{\Omega} u_{i}\left\langle\nabla\left(\left\langle\nabla x_{\alpha}, \nabla u_{i}\right\rangle+x_{\alpha} \Delta u_{i}\right), \nabla x_{\alpha}\right\rangle \\
& =-\left\|\left\langle\nabla u_{i}, \nabla x_{\alpha}\right\rangle\right\|^{2}-\int_{\Omega}\left\langle\nabla\left(x_{\alpha} u_{i}\right), \nabla\left\langle\nabla u_{i}, \nabla x_{\alpha}\right\rangle\right\rangle-\int_{\Omega} u_{i}\left\langle\nabla\left(x_{\alpha} \Delta u_{i}\right), \nabla x_{\alpha}\right\rangle \\
& =-\left\|\left\langle\nabla u_{i}, \nabla x_{\alpha}\right\rangle\right\|^{2}+\int_{\Omega}\left\langle\nabla u_{i}, \nabla x_{\alpha}\right\rangle \Delta\left(x_{\alpha} u_{i}\right)+\int_{\Omega} x_{\alpha} \Delta u_{i}\left\langle\nabla u_{i}, \nabla x_{\alpha}\right\rangle \\
& =\left\|\left\langle\nabla u_{i}, \nabla x_{\alpha}\right\rangle\right\|^{2}+2 \int_{\Omega} x_{\alpha} \Delta u_{i}\left\langle\nabla u_{i}, \nabla x_{\alpha}\right\rangle \\
& =\left\|\left\langle\nabla u_{i}, \nabla x_{\alpha}\right\rangle\right\|^{2}-2 \int_{\Omega}\left\langle\nabla u_{i}, \nabla\left(x_{\alpha}\left\langle\nabla u_{i}, \nabla x_{\alpha}\right\rangle\right)\right\rangle \\
& =-\left\|\left\langle\nabla u_{i}, \nabla x_{\alpha}\right\rangle\right\|^{2}-2 \int_{\Omega} x_{\alpha}\left\langle\nabla u_{i}, \nabla\left\langle\nabla u_{i}, \nabla x_{\alpha}\right\rangle\right\rangle \\
& =-\left\|\left\langle\nabla u_{i}, \nabla x_{\alpha}\right\rangle\right\|^{2}+1 .
\end{aligned}
$$

On the other hand, for $\epsilon>0$, we have

$$
\begin{aligned}
\int_{\Omega}\left\langle\nabla x_{\alpha}, W_{\alpha i}\right\rangle \Delta u_{i} & =\int_{\Omega}\left\langle\Delta u_{i} \nabla x_{\alpha}-\nabla\left\langle\nabla u_{i}, \nabla x_{\alpha}\right\rangle, W_{\alpha i}\right\rangle \\
& \leq \frac{\epsilon}{2}\left\|W_{\alpha i}\right\|^{2}+\frac{1}{2 \epsilon}\left\|\Delta u_{i} \nabla x_{\alpha}-\nabla\left\langle\nabla u_{i}, \nabla x_{\alpha}\right\rangle\right\|^{2} .
\end{aligned}
$$

From (2.36), we have

$$
\sum_{\alpha=1}^{n} \int_{\Omega}\left\langle\nabla x_{\alpha}, W_{\alpha i}\right\rangle \Delta u_{i}=n-1
$$

Also, one can check that

$$
\sum_{\alpha=1}^{n}\left\|\Delta u_{i} \nabla x_{\alpha}-\nabla\left\langle\nabla u_{i}, \nabla x_{\alpha}\right\rangle\right\|^{2}=(n-1) \int_{\Omega} u_{i} \Delta^{2} u_{i} \leq(n-1) \Lambda_{i}^{1 /(l-1)} .
$$

Thus we have from (2.37)-(2.39) that

$$
n-1 \leq \frac{\epsilon}{2} \sum_{\alpha=1}^{n}\left\|W_{\alpha i}\right\|^{2}+\frac{n-1}{2 \epsilon} \Lambda_{i}^{1 /(l-1)} .
$$

Taking

$$
\epsilon=\sqrt{\frac{(n-1) \Lambda_{i}^{1 /(l-1)}}{\sum_{\alpha=1}^{n}\left\|W_{\alpha i}\right\|^{2}}},
$$


we get (2.35). This completes the proof of Lemma 2.3 .

Let us continue the proof of Theorem 1.1. Since $\left\|u_{i}\right\|^{2}=\left\|W_{\alpha i}\right\|^{2}+\left\|\nabla y_{\alpha i}\right\|^{2}$, we have from (2.35) that

$$
\begin{aligned}
n \Lambda_{i}\left\|u_{i}\right\|^{2} & =\Lambda_{i} \sum_{\alpha=1}^{n}\left\|W_{\alpha i}\right\|^{2}+\Lambda_{i} \sum_{\alpha=1}^{n}\left\|\nabla y_{\alpha i}\right\|^{2} \\
& \geq(n-1) \Lambda_{i}^{(l-2) /(l-1)}+\Lambda_{i} \sum_{\alpha=1}^{n}\left\|\nabla y_{\alpha i}\right\|^{2},
\end{aligned}
$$

which, combining with (2.25), implies that

$$
-\Lambda_{i} \sum_{\alpha=1}^{n}\left\|\nabla y_{\alpha i}\right\|^{2} \leq \frac{(n-2 l-2)}{3} \int_{\Omega} u_{i}(-\Delta)^{l-1} u_{i}-\frac{(n-1)}{3} \Lambda_{i}^{(l-2) /(l-1)} .
$$

Substituting (2.42) into (2.24) and using Lemma 2.1, we get

$$
\begin{aligned}
& n \sum_{i=1}^{k}\left(\Lambda_{k+1}-\Lambda_{i}\right)^{2} \\
\leq & \sum_{i=1}^{k} \delta_{i}\left(\Lambda_{k+1}-\Lambda_{i}\right)^{2}\left(\left(2 l^{2}+(n-4) l+3-n+\frac{n-2 l-2}{3}\right) \int_{\Omega} u_{i}(-\Delta)^{l-1} u_{i}-\frac{(n-1)}{3} \Lambda_{i}^{(l-2) /(l-1)}\right) \\
& +\sum_{i=1}^{k} \frac{1}{\delta_{i}}\left(\Lambda_{k+1}-\Lambda_{i}\right) \sum_{\alpha=1}^{n} \Lambda_{i}^{1 /(l-1)} \\
\leq & \sum_{i=1}^{k} \delta_{i}\left(\Lambda_{k+1}-\Lambda_{i}\right)^{2}\left(\left(2 l^{2}+(n-4) l+3-n+\frac{n-2 l-2}{3}-\frac{(n-1)}{3}\right) \Lambda_{i}^{(l-2) /(l-1)}\right) \\
& +\sum_{i=1}^{k} \frac{1}{\delta_{i}}\left(\Lambda_{k+1}-\Lambda_{i}\right) \Lambda_{i}^{1 /(l-1)} \\
= & \sum_{i=1}^{k} \delta_{i}\left(\Lambda_{k+1}-\Lambda_{i}\right)^{2}\left(2 l^{2}+\left(n-\frac{14}{3}\right) l+\frac{8}{3}-n\right) \Lambda_{i}^{(l-2) /(l-1)} \\
& +\sum_{i=1}^{k} \frac{1}{\delta_{i}}\left(\Lambda_{k+1}-\Lambda_{i}\right) \Lambda_{i}^{1 /(l-1)} .
\end{aligned}
$$

This completes the proof of Theorem 1.1.

Proof of Corollary 1.2. By induction, one can show that

$$
\begin{aligned}
& \left\{\sum_{i=1}^{k}\left(\Lambda_{k+1}-\Lambda_{i}\right)^{2} \Lambda_{i}^{(l-2) /(l-1)}\right\}\left\{\sum_{i=1}^{k}\left(\Lambda_{k+1}-\Lambda_{i}\right) \Lambda_{i}^{1 /(l-1)}\right\} \\
\leq & \left\{\sum_{i=1}^{k}\left(\Lambda_{k+1}-\Lambda_{i}\right)^{2}\right\}\left\{\sum_{i=1}^{k}\left(\Lambda_{k+1}-\Lambda_{i}\right) \Lambda_{i}\right\},
\end{aligned}
$$

which, combining with (1.12), gives (1.13).

Proof of Theorem 1.2. We use the same notations as in the beginning of this section and take $M$ to be the unit $n$-sphere $S^{n}$. Let $x_{1}, x_{2}, \cdots, x_{n+1}$ be the standard coordinate functons of the Euclidean space $\mathbf{R}^{n+1}$, then

$$
S=\left\{\left(x_{1}, \ldots, x_{n+1}\right) \in \mathbf{R}^{n+1} ; \sum_{\alpha=1}^{n+1} x_{\alpha}^{2}=1\right\} .
$$


It is well known that

$$
\Delta x_{\alpha}=-n x_{\alpha}, \quad \alpha=1, \cdots, n+1 .
$$

As in the proof of Theorem 1.1, we decompose the vector-valued functions $x_{\alpha} \nabla u_{i}$ as

$$
x_{\alpha} \nabla u_{i}=\nabla h_{\alpha i}+W_{\alpha i},
$$

where $h_{\alpha i} \in H_{l, D}^{2}(\Omega), \nabla h_{\alpha i}$ is the projection of $x_{\alpha} \nabla u_{i}$ in $\mathbf{H}_{1, D}^{2}(\Omega), W_{\alpha i} \perp \mathbf{H}_{1, D}^{2}(\Omega)$ and

$$
\left.W_{\alpha i}\right|_{\partial \Omega}=0, \operatorname{div} W_{\alpha i}=0 .
$$

We also consider the functions $\phi_{\alpha i}: \Omega \rightarrow \mathbf{R}$, given by

$$
\phi_{\alpha i}=h_{\alpha i}-\sum_{j=1}^{k} b_{\alpha i j} u_{j}, \quad b_{\alpha i j}=\int_{\Omega} x_{\alpha}\left\langle\nabla u_{i}, \nabla u_{j}\right\rangle=b_{\alpha j i} .
$$

Then

$$
\begin{gathered}
\left.\phi_{\alpha i}\right|_{\partial \Omega}=\left.\frac{\partial \phi_{\alpha i}}{\partial \nu}\right|_{\partial \Omega}=\left.\cdots \frac{\partial^{l-1} \phi_{\alpha i}}{\partial \nu^{l-1}}\right|_{\partial \Omega}=0 \\
\left(\phi_{\alpha i}, u_{j}\right)_{D}=\int_{\Omega}\left\langle\nabla \phi_{\alpha i}, \nabla u_{j}\right\rangle=0, \quad \forall j=1, \cdots, k
\end{gathered}
$$

and we have the basic Rayleigh-Ritz inequality for $\Lambda_{k+1}$ :

$$
\Lambda_{k+1} \int_{\Omega}\left|\nabla \phi_{\alpha i}\right|^{2} \leq \int_{D} \phi_{\alpha i}(-\Delta)^{l} \phi_{\alpha i}, \quad \forall \alpha=1, \cdots, n, \quad i=1, \cdots, k .
$$

We have

$$
\Delta \phi_{\alpha i}=\left\langle\nabla x_{\alpha}, \nabla u_{i}\right\rangle+x_{\alpha} \Delta u_{i}-\sum_{j=1}^{k} b_{\alpha i j} \Delta u_{j}
$$

and from $(2.56)$ in [28],

$$
\begin{aligned}
& \int_{\Omega} \phi_{\alpha i}(-\Delta)^{l} \phi_{\alpha i} \\
& =\int_{\Omega}(-1)^{l}\left(\left\langle\nabla x_{\alpha}, \nabla u_{i}\right\rangle+x_{\alpha} \Delta u_{i}\right) \Delta^{l-2}\left(\left\langle\nabla x_{\alpha}, \nabla u_{i}\right\rangle+x_{\alpha} \Delta u_{i}\right)-\sum_{j=1}^{k} \Lambda_{j} b_{\alpha i j}^{2} .
\end{aligned}
$$

For a function $g$ on $\Omega$, we have (cf. (2.31) in [34])

$$
\Delta\left\langle\nabla x_{\alpha}, \nabla g\right\rangle=-2 x_{\alpha} \Delta g+\left\langle\nabla x_{\alpha}, \nabla((\Delta+n-2) g)\right\rangle .
$$

For each $q=0,1, \cdots$, thanks to (2.43) and (2.50), there are polynomials $F_{q}$ and $G_{q}$ of degree $q$ such that

$$
\Delta^{q}\left(\left\langle\nabla x_{\alpha}, \nabla u_{i}\right\rangle+x_{\alpha} \Delta u_{i}\right)=x_{\alpha} F_{q}(\Delta) \Delta u_{i}+\left\langle\nabla x_{\alpha}, \nabla\left(G_{q}(\Delta) u_{i}\right)\right\rangle .
$$

It is obvious that

$$
F_{0}=1, \quad G_{0}=1 \text {. }
$$


It follows from (2.43) and (2.50) that

$$
\Delta\left(x_{\alpha} \Delta u_{i}+\left\langle\nabla x_{\alpha}, \nabla u_{i}\right\rangle\right)=x_{\alpha}(\Delta-(n+2)) \Delta u_{i}+\left\langle\nabla x_{\alpha}, \nabla\left((3 \Delta+n-2) u_{i}\right)\right\rangle,
$$

which gives

$$
F_{1}(t)=t-(n+2), \quad G_{1}(t)=3 t+n-2 .
$$

Also, when $q \geq 2$, we have (cf. (2.65) and (2.66) in [28])

$$
\begin{aligned}
& F_{q}(t)=(2 t-2) F_{q-1}(t)-\left(t^{2}+2 t-n(n-2)\right) F_{q-2}(t), \quad q=2, \cdots, \\
& G_{q}(t)=(2 t-2) G_{q-1}(t)-\left(t^{2}+2 t-n(n-2)\right) G_{q-2}(t), \quad q=2, \cdots
\end{aligned}
$$

For each $q=1,2, \cdots$, let us set

$$
\Phi_{q}(t)=t F_{q-1}(t)-G_{q-1}(t) .
$$

We conclude from (2.52), (2.54)-(2.56) that the polynomials $\Phi_{q}, q=1,2, \cdots$, are defined inductively by (1.15) and (1.16). Substituting

$$
\Delta^{l-2}\left(\left\langle\nabla x_{\alpha}, \nabla u_{i}\right\rangle+x_{\alpha} \Delta u_{i}\right)=x_{\alpha} F_{l-2}(\Delta) \Delta u_{i}+\left\langle\nabla x_{\alpha}, \nabla\left(G_{l-2}(\Delta) u_{i}\right)\right\rangle
$$

into (2.49), we get

$$
\begin{aligned}
& \int_{\Omega} \phi_{\alpha i}(-\Delta)^{l} \phi_{\alpha i} \\
= & \int_{\Omega}(-1)^{l}\left(\left\langle\nabla x_{\alpha}, \nabla u_{i}\right\rangle\left\langle\nabla x_{\alpha}, \nabla\left(G_{l-2}(\Delta) u_{i}\right)\right\rangle+\left\langle x_{\alpha} \nabla x_{\alpha}, \Delta u_{i} \nabla\left(G_{l-2}(\Delta) u_{i}\right)+\left(F_{l-2}(\Delta) \Delta u_{i}\right) \nabla u_{i}\right\rangle\right) \\
& +\int_{\Omega}(-1)^{l} x_{\alpha}^{2} \Delta u_{i} F_{l-2}(\Delta)\left(\Delta u_{i}\right)-\sum_{j=1}^{k} \Lambda_{j} b_{\alpha i j}^{2} .
\end{aligned}
$$

Summing over $\alpha$ and noticing

$$
\sum_{\alpha=1}^{n+1} x_{\alpha}^{2}=1, \quad \sum_{\alpha=1}^{n+1}\left\langle\nabla x_{\alpha}, \nabla u_{i}\right\rangle\left\langle\nabla x_{\alpha}, \nabla\left(G_{l-2}(\Delta) u_{i}\right)\right\rangle=\left\langle\nabla u_{i}, \nabla\left(G_{l-2}(\Delta) u_{i}\right)\right\rangle,
$$

we infer

$$
\begin{aligned}
& \sum_{\alpha=1}^{n+1} \int_{\Omega} \phi_{\alpha i}(-\Delta)^{l} \phi_{\alpha i} \\
= & \int_{\Omega}(-1)^{l}\left\langle\nabla u_{i}, \nabla\left(G_{l-2}(\Delta) u_{i}\right)\right\rangle+\int_{\Omega}(-1)^{l} \Delta u_{i} F_{l-2}(\Delta)\left(\Delta u_{i}\right)-\sum_{\alpha=1}^{n+1} \sum_{j=1}^{k} \Lambda_{j} b_{\alpha i j}^{2} \\
= & \int_{\Omega}(-1)^{l-1} u_{i} \Delta\left(G_{l-2}(\Delta) u_{i}\right)+\int_{\Omega}(-1)^{l} u_{i} \Delta\left(F_{l-2}(\Delta)\left(\Delta u_{i}\right)\right)-\sum_{\alpha=1}^{n+1} \sum_{j=1}^{k} \Lambda_{j} b_{\alpha i j}^{2} \\
= & \int_{\Omega}(-1)^{l} u_{i}\left(\Delta F_{l-2}(\Delta)-G_{l-2}(\Delta)\right)\left(\Delta u_{i}\right)-\sum_{\alpha=1}^{n+1} \sum_{j=1}^{k} \Lambda_{j} b_{\alpha i j}^{2} \\
= & \int_{\Omega}(-1)^{l} u_{i} \Phi_{l-1}(\Delta)\left(\Delta u_{i}\right)-\sum_{\alpha=1}^{n+1} \sum_{j=1}^{k} \Lambda_{j} b_{\alpha i j}^{2}
\end{aligned}
$$




$$
\begin{aligned}
& =\int_{\Omega}(-1)^{l} u_{i}\left(\Delta^{l-1}-a_{l-2} \Delta^{l-2}+\cdots+(-1)^{l-2} a_{1} \Delta-(n-2)^{l-2}\right)\left(\Delta u_{i}\right)-\sum_{\alpha=1}^{n+1} \sum_{j=1}^{k} \Lambda_{j} b_{\alpha i j}^{2} \\
& =\Lambda_{i}+(-1)^{l}(n-2)^{l-2}+\sum_{j=1}^{l-2} a_{j} \int_{\Omega} u_{i}(-\Delta)^{j+1} u_{i}-\sum_{\alpha=1}^{n+1} \sum_{j=1}^{k} \Lambda_{j} b_{\alpha i j}^{2} .
\end{aligned}
$$

Set

$$
H_{i}=(-1)^{l}(n-2)^{l-2}+\sum_{j=1}^{l-2} a_{j}^{+} \Lambda_{i}^{j /(l-1)}
$$

then it is easy to check from Lemma 2.1 that

$$
(-1)^{l}(n-2)^{l-2}+\sum_{j=0}^{l-2} a_{j} \int_{\Omega} u_{i}(-\Delta)^{j+1} u_{i} \leq H_{i} .
$$

Substituting (2.62) into (2.60), we have

$$
\sum_{\alpha=1}^{n+1} \int_{\Omega} \phi_{\alpha i}(-\Delta)^{l} \phi_{\alpha i} \leq \Lambda_{i}+H_{i}-\sum_{\alpha=1}^{n+1} \sum_{j=1}^{k} \Lambda_{j} b_{\alpha i j}^{2} .
$$

Observe from (2.44) and (2.46) that

$$
\left\|x_{\alpha} \nabla u_{i}\right\|^{2}=\left\|\nabla h_{\alpha i}\right\|^{2}+\left\|W_{\alpha i}\right\|^{2}=\left\|\nabla \phi_{\alpha i}\right\|^{2}+\left\|W_{\alpha i}\right\|^{2}+\sum_{j=1}^{k} b_{\alpha i j}^{2} .
$$

Summing over $\alpha$, one gets

$$
1=\sum_{\alpha=1}^{n+1}\left(\left\|\nabla \phi_{\alpha i}\right\|^{2}+\left\|W_{\alpha i}\right\|^{2}+\sum_{j=1}^{k} b_{\alpha i j}^{2}\right) .
$$

Combining (2.47), (2.63) and (2.65), we get

$$
\sum_{\alpha=1}^{n+1}\left(\Lambda_{k+1}-\Lambda_{i}\right)\left\|\nabla \phi_{\alpha i}\right\|^{2} \leq H_{i}+\sum_{\alpha=1}^{n+1} \Lambda_{i}\left\|W_{\alpha i}\right\|^{2}+\sum_{\alpha=1}^{n+1} \sum_{j=1}^{k}\left(\Lambda_{i}-\Lambda_{j}\right) b_{\alpha i j}^{2} .
$$

Set

$$
Z_{\alpha i}=\nabla\left\langle\nabla x_{\alpha}, \nabla u_{i}\right\rangle-\frac{n-2}{2} x_{\alpha} \nabla u_{i}, \quad c_{\alpha i j}=\int_{\Omega}\left\langle\nabla u_{j}, Z_{\alpha i}\right\rangle ;
$$

then $c_{\alpha i j}=-c_{\alpha j i}$ (cf. Lemma in [34]). By using the same arguments as in the proof of (2.37) in [34], we have

$$
\begin{aligned}
& \left(\Lambda_{k+1}-\Lambda_{i}\right)^{2}\left(2\left\|\left\langle\nabla x_{\alpha}, \nabla u_{i}\right\rangle\right\|^{2}+\int_{\Omega}\left\langle\nabla x_{\alpha}^{2}, \Delta u_{i} \nabla u_{i}\right\rangle+(n-2)\left\|x_{\alpha} \nabla u_{i}\right\|^{2}+2 \sum_{j=1}^{k} b_{\alpha i j} c_{\alpha i j}\right) \\
\leq & \delta_{i}\left(\Lambda_{k+1}-\Lambda_{i}\right)^{3}\left\|\nabla \phi_{\alpha i}\right\|^{2}+\frac{\Lambda_{k+1}-\Lambda_{i}}{\delta_{i}}\left(\left\|Z_{\alpha i}\right\|^{2}-\sum_{j=1}^{k} c_{\alpha i j}^{2}\right)+(n-2)\left(\Lambda_{k+1}-\Lambda_{i}\right)^{2}\left\|W_{\alpha i}\right\|^{2} .
\end{aligned}
$$


Since

$$
\sum_{\alpha=1}^{n+1}\left\|\left\langle\nabla x_{\alpha}, \nabla u_{i}\right\rangle\right\|^{2}=\int_{\Omega}\left|\nabla u_{i}\right|^{2}=1,
$$

we have by summing over $\alpha$ in (2.68) from 1 to $n+1$ that

$$
\begin{aligned}
& \left(\Lambda_{k+1}-\Lambda_{i}\right)^{2}\left(n+2 \sum_{\alpha=1}^{n+1} \sum_{j=1}^{k} b_{\alpha i j} c_{\alpha i j}\right) \\
\leq & \delta_{i} \sum_{\alpha=1}^{n+1}\left(\Lambda_{k+1}-\Lambda_{i}\right)^{3}\left\|\nabla \phi_{\alpha i}\right\|^{2}+\frac{\Lambda_{k+1}-\Lambda_{i}}{\delta_{i}} \sum_{\alpha=1}^{n+1}\left(\left\|Z_{\alpha i}\right\|^{2}-\sum_{j=1}^{k} c_{\alpha i j}^{2}\right) \\
& +(n-2) \sum_{\alpha=1}^{n+1}\left(\Lambda_{k+1}-\Lambda_{i}\right)^{2}\left\|W_{\alpha i}\right\|^{2} .
\end{aligned}
$$

From (2.77), (2.78) and (2.80) in [28], we have

$$
\Lambda_{i}^{1 /(l-1)}-(n-2)>0,
$$

and

$$
\sum_{\alpha=1}^{n+1}\left\|W_{\alpha i}\right\|^{2} \leq 1-\frac{1}{\Lambda_{i}^{1 /(l-1)}-(n-2)} .
$$

We have by combining (2.66), (2.70), (2.72) and (2.73) that

$$
\begin{aligned}
& \left(\Lambda_{k+1}-\Lambda_{i}\right)^{2}\left(n+2 \sum_{\alpha=1}^{n+1} \sum_{j=1}^{k} b_{\alpha i j} c_{\alpha i j}\right) \\
\leq & \delta_{i}\left(\Lambda_{k+1}-\Lambda_{i}\right)^{2}\left(H_{i}+\sum_{\alpha=1}^{n+1} \sum_{j=1}^{k}\left(\Lambda_{i}-\Lambda_{j}\right) b_{\alpha i j}^{2}\right) \\
& +\frac{\Lambda_{k+1}-\Lambda_{i}}{\delta_{i}}\left(\left\|Z_{\alpha i}\right\|^{2}-\sum_{\alpha=1}^{n+1} \sum_{j=1}^{k} c_{\alpha i j}^{2}\right)+\sum_{\alpha=1}^{n+1}\left(\Lambda_{k+1}-\Lambda_{i}\right)^{2}\left(\delta_{i} \Lambda_{i}+n-2\right)\left\|W_{\alpha i}\right\|^{2} \\
\leq & \delta_{i}\left(\Lambda_{k+1}-\Lambda_{i}\right)^{2}\left(H_{i}+\sum_{\alpha=1}^{n+1} \sum_{j=1}^{k}\left(\Lambda_{i}-\Lambda_{j}\right) b_{\alpha i j}^{2}\right) \\
& +\frac{\Lambda_{k+1}-\Lambda_{i}}{\delta_{i}}\left(\left(\Lambda_{i}^{1 /(l-1)}+\frac{(n-2)^{2}}{4}\right)-\sum_{\alpha=1}^{n+1} \sum_{j=1}^{k} c_{\alpha i j}^{2}\right) \\
& +\left(\Lambda_{k+1}-\Lambda_{i}\right)^{2}\left(\delta_{i} \Lambda_{i}+n-2\right)\left(1-\frac{1}{\Lambda_{i}^{1 /(l-1)}-(n-2)}\right) .
\end{aligned}
$$


Since $\left\{\delta_{i}\right\}_{i=1}^{k}$ is a positive non-increasing monotone sequence, we have

$$
\begin{aligned}
& 2 \sum_{\alpha=1}^{n+1} \sum_{i, j=1}^{k}\left(\Lambda_{k+1}-\Lambda_{i}\right)^{2} b_{\alpha i j} c_{\alpha i j} \\
\geq & \sum_{\alpha=1}^{n+1} \sum_{i, j=1}^{k} \delta_{i}\left(\Lambda_{k+1}-\Lambda_{i}\right)^{2}\left(\Lambda_{i}-\Lambda_{j}\right) b_{\alpha i j}^{2}-\sum_{\alpha=1}^{n+1} \sum_{i, j=1}^{k} \frac{\Lambda_{k+1}-\Lambda_{i}}{\delta_{i}} c_{\alpha i j}^{2} .
\end{aligned}
$$

Hence, by summing over $i$ from 1 to $k$ in (2.74), we infer

$$
\begin{aligned}
& n \sum_{i=1}^{k}\left(\Lambda_{k+1}-\Lambda_{i}\right)^{2} \\
\leq & \sum_{i=1}^{k}\left(\Lambda_{k+1}-\Lambda_{i}\right)^{2}\left(\delta_{i} H_{i}+\left(\delta_{i} \Lambda_{i}+n-2\right)\left(1-\frac{1}{\Lambda_{i}^{1 /(l-1)}-(n-2)}\right)\right) \\
& +\sum_{i=1}^{k} \frac{\Lambda_{k+1}-\Lambda_{i}}{\delta_{i}}\left(\Lambda_{i}^{1 /(l-1)}+\frac{(n-2)^{2}}{4}\right) .
\end{aligned}
$$

That is

$$
\begin{aligned}
& \sum_{i=1}^{k}\left(\Lambda_{k+1}-\Lambda_{i}\right)^{2}\left(2+\frac{n-2}{\Lambda_{i}^{1 /(l-1)}-(n-2)}\right) \\
\leq & \sum_{i=1}^{k}\left(\Lambda_{k+1}-\Lambda_{i}\right)^{2} \delta_{i}\left(H_{i}+\Lambda_{i}\left(1-\frac{1}{\Lambda_{i}^{1 /(l-1)}-(n-2)}\right)\right) \\
& +\sum_{i=1}^{k} \frac{\left(\Lambda_{k+1}-\Lambda_{i}\right)}{\delta_{i}}\left(\Lambda_{i}^{1 /(l-1)}+\frac{(n-2)^{2}}{4}\right) \\
= & \sum_{i=1}^{k}\left(\Lambda_{k+1}-\Lambda_{i}\right)^{2} \delta_{i} S_{i}+\sum_{i=1}^{k} \frac{\left(\Lambda_{k+1}-\Lambda_{i}\right)}{\delta_{i}}\left(\Lambda_{i}^{1 /(l-1)}+\frac{(n-2)^{2}}{4}\right),
\end{aligned}
$$

where $S_{i}$ is given by (1.19). This completes the proof of Theorem 1.2.

\section{References}

[1] M. S. Ashbaugh, Isoperimetric and universal inequalities for eigenvalues, in Spectral theory and geometry (Edingurgh, 1998), E. B. Davies and Yu Safarov eds., London Math. Soc. Lecture Notes, vol. 273, Cambridge Univ. Press, cambridge, 1999, pp. 95-139.

[2] M. S. Ashbaugh, The universal eigenvalue bounds of Payne-Pólya-Weinberger, Hile-Protter and H C Yang, Proc. India Acad. Sci. Math. Sci. 112 (2002), 3-30.

[3] M. S. Ashbaugh and L. Hermi, A unified approach to universal inequalities for eigenvalues of elliptic operators, Pacific J. Math. 217 (2004), 201-219.

[4] M. S. Ashbaugh and L. Hermi, On Harrell-Stubbe type inequalities for the discrete spectrum of a self-adjoint operator, http://arxiv.org/abs/0712.4396.

[5] D. Chen and Q. -M. Cheng, Extrinsic estimates for eigenvalues of the Laplace operator, J. Math. Soc. Japan 60 (2008), 325-339. 
[6] Q. -M. Cheng, T. Ichikawa and S. Mametsuka, Inequalities for eigenvalues of Laplacian with any order, Commun. Contemp. Math. 11 (2009), 639-655.

[7] Q. -M. Cheng, T. Ichikawa and S. Mametsuka, Estimates for eigenvalues of the poly-Laplacian with any order in a unit sphere, Calc. Var. Partial Differential Equations 36 (2009), 507-523.

[8] Q. - M. Cheng, T. Ichikawa and S. Mametsuka, Estimates for eigenvalues of a clamped plate problem on Riemannian manifolds, J. Math. Soc. Japan 62 (2010),673-686.

[9] Q. -M. Cheng and H. C. Yang, Estimates on eigenvalues of Laplacian, Math. Ann. 331 (2005), 445-460.

[10] Q. -M. Cheng and H. C. Yang, Inequalities for eigenvalues of a clamped plate problem, Trans. Amer. Math. Soc. 358 (2006), 2625-2635.

[11] Q. -M. Cheng and H. C. Yang, Universal bounds for eigenvalues of a buckling problem, Comm. Math. Phys. 262 (2006), 663-675.

[12] Q. -M. Cheng and H. C. Yang, Inequalities for eigenvalues of Laplacian on domains and compact complex hypersurfaces in complex projective spaces, J. Math. Soc. Japan 58 (2006), 545-561.

[13] Q. -M. Cheng and H. C. Yang, Bounds on eigenvalues of Dirichlet Laplacian, Math. Ann. 337 (2007), 159-175.

[14] Q. -M. Cheng and H. C. Yang, Estimates for eigenvalues on Riemannian manifolds, J. Differential Equations 247 (2009), no. 8, 2270-2281.

[15] Q. -M. Cheng and H. C. Yang, Universal inequalities for eigenvalues of a system of elliptic equations. Proc. Roy. Soc. Edinburgh Sect. A 139 (2009), 273-285.

[16] Q. -M. Cheng and H. C. Yang, Universal bounds for eigenvalues of a buckling problem II, preprint, 2009.

[17] M. P. do Carmo, Q. Wang and C. Xia, Inequalities for eigenvalues of elliptic operators in divergence form on Riemannian manifolds, Ann. Mat. Pura Appl. 189 (2010), 643-660.

[18] E. M. Harrell, Some geometric bounds on eigenvalue gaps, Comm. Partial Differential Equations 18 (1993), 179-198.

[19] E. M. Harrell, L. Hermi, Differential inequalities for Riesz means and Weyl-type bounds for eigenvalues, J. Funct. Anal. 254 (2008), 3173-3191.

[20] E. M. Harrell and P. L. Michel, Commutator bounds for eigenvalues, with applications to spectral geometry, Comm. Partial Differential Equations 19 (1994), 2037-2055.

[21] E. M. Harrell and P. L. Michel, Commutator bounds for eigenvalues of some differential operators, Lecture Notes in Pure and Applied Mathematics, vol. 168(eds.) G Ferreyra, G R Goldstein and F Neubrander (New York: Marcel Dekker) (1995) pp. 235-244.

[22] E. M. Harrell and S. Yildirim Yolcu, Eigenvalue inequalities for Klein-Gordon operators, J. Funct. Anal. 256 (2009), 3977-3995.

[23] E. M. Harrell and J. Stubbe, On trace identities and the universal eigenvalue estimates for some partial differential operators, Trans. Amer. Math. Soc. 349 (1997), 1797-1809.

[24] E. Hebey, Nonlinear analysis on manifolds: Sobolev spaces and inequalities. Courant Lecture Notes in Mathematics, 5. New York University, Courant Institute of Mathematical Sciences, New York; American Mathematical Society, Providence, RI, 1999. x+309 pp. 
[25] G. N. Hile and M. H. Protter, Inequalities for eigenvalues of the Laplacian, Indiana Univ. Math. J. 29 (1980), 523-538.

[26] G. N. Hile and R. Z. Yeh, Inequalities for eigenvalues of the biharmonic operator, Pacific J. Math. 112 (1984), 115-133.

[27] S. Ilias and O. Makhoul, 'Universal' inequalities for the eigenvalues of the Hodge de Rham Laplacian, Ann. Global Anal. Geom. 36 (2009), 191-204.

[28] J. Jost, X. Li-Jost, Q. Wang and C. Xia, Universal inequalities for eigenvalues of the buckling problem of arbitrary order, Comm. Partial Differential Equations 35 (2010), 1563-1589.

[29] J. Jost, X. Li-Jost, Q. Wang and C. Xia, Universal bounds for eigenvalues of the polyharmonic operator, to appear in Trans. Amer. Math. Soc.

[30] L. E. Payne, G. Pólya and H. F. Weinberger, sur le quotient de deux fréquences propres cosécutives, Comptes Rendus Acad. Sci. Paris 241 (1955), 917-919.

[31] L. E. Payne, G. Pólya and H. F. Weinberger, On the ratio of consecutive eigenvalues, J. Math. and Phys. 35 (1956), 289-298.

[32] H. Sun, Q. -M. Cheng and H. C. Yang, Lower order eigenvalues of Dirichlet Laplacian, Manuscripta Math. 125 (2008), 139-156.

[33] Q. Wang, C. Xia, Universal bounds for eigenvalues of the biharmonic operator on Riemannian manifolds, J. Funct. Anal. 245 (2007), 334-352.

[34] Q. Wang, C. Xia, Universal bounds for eigenvalues of the buckling problem on spherical domains, Comm. Math. Phys. 270 (2007), 759-775.

[35] Q. Wang, C. Xia, Universal bounds for eigenvalues of Schrödinger operator on Riemannian manifolds, Ann. Acad. Sci. Fenn. Math. 33 (2008), 319-336.

[36] Q. Wang, C. Xia, Universal bounds for eigenvalues of the biharmonic operator, J. Math. Anal. Appl. 364 (2010), 1-17.

[37] Q. Wang, C. Xia, Inequalities for eigenvalues of the biharmonic operator with weight on Riemannian manifolds. J. Math. Soc. Japan, 62 (2010), 597-622.

[38] Q. Wang, C. Xia, Inequalities for eigenvalues of a clamped plate problem. Calc. Var. Partial Differential Equations 2010. DOI:10.1007/s00526-010-0340-4.

[39] H. C. Yang, An estimate of the difference between consecutive eigenvalues, preprint IC/91/60 of ICTP, Trieste, 1991.

Qing-Ming Cheng (cheng@ms.saga-u.ac.jp) and Xuerong Qi (qixuerong609@gmail.com)

Department of Mathematics

Faculty of Science and Engineering

Saga University

Saga 840-8502, Japan

Qiaoling Wang (wang@mat.unb.br) and Changyu Xia (xia@mat.unb.br)

Departamento de Matemática

Universidade de Brasília

70910-900-Brasília-DF, Brazil 